\title{
A New Group Fuzzy-Delphi Analytic Hierarchy Process Method with Its Application in Enterprise Intellectual Property Financing Decision Analysis
}

\author{
Bo FENG ${ }^{\mathrm{a}, \mathrm{b}}$, Zhipeng HUI ${ }^{\mathrm{a}}$, Junwen FENG ${ }^{\mathrm{a}, \mathrm{b}, \mathrm{1}}$ \\ ${ }^{\mathrm{a}}$ Nanjing University of Science and Technology, 210094, China

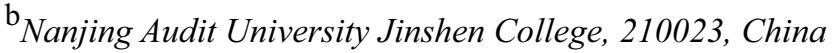

\begin{abstract}
The traditional analytic hierarchy process (AHP), fuzzy evaluation method and the Delphi method of group decision-making are organically combined and a new method of system analysis called Fuzzy Delphi Analytic Hierarchy Process (FDAHP) is proposed. Based on the Delphi survey of the group decisionmaking information, the group's pairwise judgment of objects is fuzzily processed, and the results of the group's overall judgment are used as decision-making environmental parameters. Furthermore, the group's comprehensive weight of objects is determined according to the optimistic coefficient of group decisionmaking consideration. A simple example is given to illustrate the specific implementation steps and feasibility of the method. Finally, the advantages and disadvantages of the method are briefly discussed, and the possible research topics in the future are proposed.
\end{abstract}

Keywords. Analytic hierarchy process; Fuzzy evaluation; Delphi analysis; Determination of weights; Sorting, Fuzzy Delphi analytic hierarchy process

\section{Introduction}

Analytic Hierarchy Process (AHP) is a systematic decision analysis method combining both qualitative analysis and quantitative analysis, which was put forward by T. L. Saaty, an outstanding American operations researcher and professor of Business School, University of Pittsburgh in the early 1970s, saw Saaty (1980) [1] and Wang (1990) [2]. Analytic hierarchy process is a kind of multi-objective group decision-making thought and method in essence. In the practical application of group decision making method, there are two ways to deal with the pairwise judgment matrix of the group decision information. One is to first determine the individual weight according to the individual judgment matrix and then synthesize the individual weight; the other is to first synthesize the individual judgment matrix and then determine the weight of the group decision information. In recent years, scholars such as Corvin (2021) [3] , Li (2012) [4], Zhou (2020) [5], Wang (2006) [6], have been improving the analytic hierarchy process to make it more convenient to use, such as establishing the order relationship between indicators, multi-attribute variable weight decision making methods, but these methods are

${ }^{1}$ Corresponding Author, Nanjing University of Science and Technology, Nanjing, China; E-mail: 313472714@qq.com 
artificially adjusted to meet the consistency of the judgment matrix, this adjustment will lead to the original discriminant information is tampered with, the reliability of the conclusion is reduced. In the early stage, aiming at the difficult problems of consistency test in AHP, some researchers such as Li (2012) [3], Lu (2002) [7], Qin (2021) [8], Zhang (2000) [9] also proposed the method of using fuzzy consistency matrix to solve them, and formed the fuzzy analytic hierarchy process. Some of them put forward the idea and principle of fuzzy analytic hierarchy process from fuzzy number. Based on these improvements, analytic hierarchy process has a more mature application in many fields, but one problem is that after experts put forward judgment matrix, weight synthesis and so on seem to be the matter of decision analysts, who play the role of half decision makers. Another problem is that these so-called fuzzy analytic hierarchy process is only fuzzy in some steps of the traditional analytic hierarchy process. In particular, the calculated weight is still certain, such as mentioned by Singh (2021) [10], Tang (2002) [11], Tao (2002) [12]. Without fuzziness, it is difficult to reflect the participation of decision makers in the later stage of weight determination. As for the consistency of the expert judgment matrix, the traditional analytic hierarchy process (AHP) requires consistency test. In fact, from the perspective of behavioral decision analysis, decision-makers should be allowed to have large inconsistencies, because inconsistencies also reflect a kind of decision-making behavior. If the process of weight determination is regulated artificially by revising the judgment range matrix, it will affect the decision judgment formed by the decision maker before.

This paper presents a decision-making method which can fully involve decision makers in weight determination and analysis. The basic idea is to form a group fuzzy pairwise judgment range matrix by synthesizing the definite pairwise judgment range matrix given by various experts in the form of fuzzy triangular number, Then, according to the properties of fuzzy trigonometric numbers and some operation methods, the fuzzy weight vector of fuzzy judgment matrix of the group is determined. The fuzzy weight vector is processed according to the thought of group decision making, and then an interactive weight vector decision analysis process is formed, and finally the decision maker is satisfied with the weight vector of group decision. This process of decision interaction can be carried out under any single criterion in the hierarchy.

\section{Basic approaches of fuzzy Delphi analytic hierarchy process}

The hierarchical structure of the problem is established, and the groups have given their pairwise judgment matrices according to the traditional analytic hierarchy process. We assume that the weight determination of the corresponding decision scheme under a certain criterion is considered. Assuming that the relative importance of $i$ and $j$ elements in the sub-level of the kth expert has been determined as $B_{i j k}$ under a certain criterion by expert investigation, forming the pairwise comparisons judgment matrix $B(k)=\left(B_{i j k}\right)$ of the $k$ th expert. Assuming that there are $m$ decision schemes or evaluation indexes to be considered, the purpose is to determine the decision weight of the $\mathrm{m}$ decision schemes on the decision criterion. The specific steps are as follows:

Step 1: Building Fuzzy Judgment Matrix of Population

The pairwise comparison judgment matrix contains the opinions of experts involved in decision making, and the judgment of relative importance is uncertain. In this method, the fuzzy triangular number is used to integrate the opinions of experts, in order to 
establish a more objective fuzzy group judgment matrix on the basis of the subjective opinions of decision makers. The pairwise judgment matrix of the group represented by triangular fuzzy number is as follows:

$\mathrm{B}=\left(\mathrm{B}_{\mathrm{ij}}\right)$, where $\mathrm{B}_{\mathrm{ij}}$ is triangular fuzzy number, which is determined by the following method:

$B_{i j}=\left[T_{i j}, U_{i j}, V_{i j}\right], T_{i j} \leq U_{i j} \leq V_{i j}, \& T_{i j}, U_{i j}, V_{i j} \in[1 / 9,1] \cup[1,9], B_{i j}$ is triangular fuzzy number; $\mathrm{T}_{\mathrm{ij}}=\min _{\mathrm{k}}\left(\mathrm{B}_{\mathrm{ijk}}\right)$.

$\mathrm{U}_{\mathrm{ij}}=$ Geomean $_{\mathrm{k}}\left(\mathrm{B}_{\mathrm{ijk}}\right)$, Here Geomean denotes geometric mean, and can also consider the weight of each expert using weighted geometric mean.

$$
\mathrm{V}_{\mathrm{ij}}=\max _{\mathrm{k}}\left(\mathrm{B}_{\mathrm{ijk}}\right)
$$

Step 2: Determining Group Fuzzy Weight Vector

Based on the group fuzzy judgment matrix $B=\left(B_{i j}\right)$, the corresponding fuzzy weight vector is determined by the column vector geometric average method as follows :

For any $\mathrm{j}, \mathrm{j}=1,2 \ldots, \mathrm{m}$, to calculate $r_{j}=\left(B_{1 j} \cdot B_{2 j} \cdot \cdots \cdot B_{m j}\right)^{1 / m}$, Where $\cdot$ denotes the product operation relation between fuzzy triangular numbers. Further standardize $r_{j}$ to $\mathrm{w}_{\mathrm{j}}$ :

$$
w_{j}=r_{j} /\left(r_{1}+r_{2}+\cdots+r_{m}\right), j=1,2, \cdots, m
$$

Step 3: Single- weight decision analysis

Firstly, the concept of cut sets in fuzzy analysis is used to defuzzify the weights, let $\mathrm{T} \in[0,1]$ be a truncated parameter, assuming $\mathrm{w}_{\mathrm{i}}=\left(\mathrm{w}_{\mathrm{i}}^{\mathrm{L}}, \mathrm{w}_{\mathrm{i}}^{\mathrm{M}}, \mathrm{w}_{\mathrm{i}}^{\mathrm{U}}\right)$,

$$
\begin{aligned}
& w_{i}^{\mathrm{L}}(T)=\left(w_{i}^{M}-w_{i}^{L}\right) T+w_{i}^{L} \\
& w_{i}^{U}(T)=\left(w_{i}^{U}-w_{i}^{M}\right) T+w_{i}^{M} \\
& (T, \lambda)=\lambda w_{i}^{U}(T)+(1-\lambda) w_{i}^{L}(T)
\end{aligned}
$$

Where $\lambda$ is the optimistic coefficient of weight for decision makers. Further, $w_{i}(T, \lambda)$ is normalized to get the normalized weight vector:

$$
(\mathrm{T}, \lambda)=\mathrm{w}_{\mathrm{i}}(\mathrm{T}, \lambda) /\left(\sum_{\mathrm{i}} \mathrm{w}_{\mathrm{i}}(\mathrm{T}, \lambda)\right)
$$

The decision weights now depend on two parameters T and $\lambda$. They mean:

The weight of the change degree of decision-making expert's judgment is reflected by $\mathrm{T}$. When $\mathrm{T}=0$, the comprehensive weight contains the decision-making weight information of each expert, and the change range of decision-making is the largest. When $\mathrm{T}=1$, the comprehensive weight contains the least decision-making weight information of each expert, which is actually equivalent to the synthesis method of expert decisionmaking weight without fuzzification. Therefore, $\mathrm{T}$ is a decision-making environmental parameter. 
$\lambda$ represents a parameter that integrates the decision-making weights of decision makers. When $\lambda=0$, the opinions of experts take the upper limit of the weights, and the experts are the most optimistic. When $\lambda=1$, experts take a conservative attitude, and take the lower limit of their weights. Therefore, $\lambda$ can be called the decision-making optimism coefficient.

In the process of determining the actual weight, if the expert group has a high consensus on the problem, it can choose a larger $\mathrm{T}$, otherwise it can choose a smaller $\mathrm{T}$. The choice of $\lambda$ reflects the degree of optimism of decision makers in evaluating the problem. The more optimistic it is, the larger its corresponding value.

\section{Operational treatments in fuzzy Delphi analytic hierarchy process}

Let $a=\left[a_{1}, a_{2}, a_{3}\right]$ and $b=\left[b_{1}, b_{2}, b_{3}\right]$ be two triangular fuzzy numbers, $T$ is any positive real number, then according to the triangular fuzzy number theory, there are the following operational properties:

$$
\begin{gathered}
a \cdot b=\left[a_{1}+b_{1}, a_{2}+b_{2}, a_{3}+b_{3}\right] \\
T_{a}=\left[T_{a 1}, T_{a 2}, T_{a 3}\right] \\
a \cdot b=\left[a_{1} b_{1}, a_{2} b_{2}, a_{3} b_{3}\right] \\
1 / a=a^{-1}=\left[1 / a_{3}, 1 / a_{2}, 1 / a_{1}\right]
\end{gathered}
$$

The standardized vector of $\mathrm{a}$ is $\mathrm{w}, \mathrm{w}$ is a fuzzy triangular number, $\mathrm{w}=\left(\mathrm{w}_{1}, \mathrm{w}_{2}, \mathrm{w}_{3}\right)$, where $\mathrm{w}_{\mathrm{i}}=\left(\mathrm{a}_{\mathrm{i}} /\left(\mathrm{a}_{1}+\mathrm{a}_{2}+\mathrm{a}_{3}\right)\right)$.

In addition, it is easy to verify that the fuzzy group judgment matrix determined in the first step is a fuzzy antisymmetric matrix, that is, $B=\left(B_{i j}\right)$ satisfies $B_{i j}=1 / B_{j i}$. In addition, when $\mathrm{i}=\mathrm{j}, \mathrm{B}_{\mathrm{ij}}=1$.

The consistency test of the judgment matrix is not carried out in the decision-making method designed above. This is because from the perspective of behavioral decisionmaking, decision makers are allowed to make pairwise judgments that are not rational.

\section{An application in enterprise intellectual property financing decision analysis}

Consider a Decision-making problem of enterprise intellectual property financing. There are four decision strategies to be considered: (1) IP securitization, (2) IP Pledge financing, (3) IP Product Business Sharing, (4) IP franchising. Four experts, denoted as E1, E4, E2 and E3, are employed to evaluate the importance of the intellectual property financing strategies. The pairwise judgment matrices given by the experts are as follows: 


$$
\begin{aligned}
\mathrm{E} 1 & =\left[\begin{array}{cccc}
1 & 1 / 2 & 1 / 3 & 1 / 4 \\
2 & 1 & 2 / 3 & 1 / 2 \\
3 & 3 / 2 & 1 & 3 / 4 \\
4 & 2 & 4 / 3 & 1
\end{array}\right], \mathrm{E} 2=\left[\begin{array}{cccc}
1 & 2 & 2 / 3 & 1 / 2 \\
1 / 2 & 1 & 1 / 3 & 1 / 4 \\
3 / 2 & 3 & 1 & 3 / 4 \\
2 & 4 & 4 / 3 & 1
\end{array}\right] \\
\mathrm{E} 3 & =\left[\begin{array}{cccc}
1 & 1 / 3 & 1 / 2 & 1 / 4 \\
3 & 1 & 3 / 2 & 3 / 4 \\
2 & 2 / 3 & 1 & 1 / 2 \\
4 & 4 / 3 & 2 & 1
\end{array}\right], \mathrm{E} 4=\left[\begin{array}{cccc}
1 & 4 / 3 & 2 & 4 \\
3 / 4 & 1 & 3 / 2 & 3 \\
1 / 2 & 3 / 2 & 1 & 2 \\
1 / 4 & 1 / 3 & 1 / 2 & 1
\end{array}\right]
\end{aligned}
$$

Accordingly, the group fuzzy pairwise judgment matrix $B=\left(B_{i j}\right)$ of the expert group is constructed as follows:

$$
\begin{aligned}
\left(\mathrm{T}_{\mathrm{ij}}\right) & =\left[\begin{array}{llll}
1.00 & 0.33 & 0.33 & 0.25 \\
0.50 & 1.00 & 0.33 & 0.25 \\
0.50 & 0.67 & 1.00 & 0.50 \\
0.25 & 0.33 & 0.50 & 1.00
\end{array}\right],\left(\mathrm{U}_{\mathrm{ij}}\right)=\left[\begin{array}{llll}
1.00 & 0.82 & 0.69 & 0.60 \\
1.23 & 1.00 & 0.84 & 0.78 \\
1.46 & 1.46 & 1.00 & 0.80 \\
1.68 & 1.37 & 1.55 & 1.00
\end{array}\right] \\
\left(\mathrm{V}_{\mathrm{ij}}\right) & =\left[\begin{array}{llll}
1.00 & 2.00 & 2.00 & 4.00 \\
3.00 & 1.00 & 1.50 & 4.00 \\
3.00 & 3.00 & 1.00 & 2.00 \\
4.00 & 2.00 & 2.00 & 1.00
\end{array}\right]
\end{aligned}
$$

The standardized fuzzy weight vector of the population can be obtained as follows: $\mathrm{w}_{1}=(0.13,0.24,0.63), \mathrm{w}_{2}=(0.13,0.27,0.60)$ $\mathrm{w}_{3}=(0.17,0.30,0.53), \mathrm{w}_{4}=(0.13,0.32,0.55)$

Assuming that the decision-making environment parameter is $\mathrm{T}=0.5$, and the decision-making optimism coefficient is $\lambda=0.8$, we can get the normalized weight vector of group decision-making as $w=(0.24,0.25,0.25,0.26)$, which means that strategy (4) is more important and the first choice. Assuming that the decision environment parameter is $\mathrm{T}=0.2$ and the decision optimism coefficient is $\lambda=0.9$, the normalized weight vector of group decision is $\mathrm{w}=(0.23,0.25,0.26,0.26)$, which means that strategy (3) and (4) are equal important. Different choices of $T$ and $\lambda$ by decision makers will lead to different decision weights. We can also make the influence curves of decision environment parameter $T$ and decision optimism coefficient $\lambda$ on decisions weight DW on the plane. It is easy to prove that for a fixed T, the decision weight is a strictly monotonically increasing linear function of $\lambda$. Similarly, for a fixed $\lambda$, the decision weight is a strictly monotonically increasing linear function of $T$. These linear functions can be directly derived.

\section{Conclusion}

In this paper, a fuzzy group analytic hierarchy process (FAHP) is designed based on analytic hierarchy process (AHP), fuzzy evaluation principle and Delphi group decision method. Through Delphi investigation, the deterministic pairwise comparison judgment matrix of the group or expert to the evaluation object is obtained, and then the fuzzy mathematics method is used to process and analyze the group survey results, and then the environmental decision parameters and decision optimism coefficient are added to form an interactive weight decision analysis process to determine the comprehensive weight of the group to the evaluation object.

There are two approaches to group decision-making and evaluation based on analytic hierarchy process in the past literature. One is to integrate the pairwise judgment matrix of experts to form a group pairwise judgment matrix, and then determine the 
decision weight or evaluation of the group to the evaluation object. The other is to determine the decision weight or evaluation of each expert based on the pairwise judgment matrix of each expert, and then integrate the decision weight of each expert to form the decision weight of the group. The workload of the two methods is large, and these methods assume a fact that the group's decision-making can be determined according to the one-time judgment of each individual in the group. The method given in this paper is not the same. On the basis of the individual investigation in the group, when the final evaluation results are determined, the group is also required to carry out decision analysis as a whole, reflecting the idea of a democracy and the unity of the main body of the concentration. The group can correct the previous judgment that is not appropriate in the final concentration process, so that the analytic hierarchy process has truly become an interactive decision analysis tool.

This paper only discusses the problem based on a single criterion, which can be easily extended to a multi-level and multi-criteria decision-making environment. In addition, the previous common group decision-making methods based on analytic hierarchy process are special cases in this paper under different combinations of decision-making environment parameters and decision-making optimism coefficients. In addition, this paper applies fuzzy triangular numbers to the fuzzy processing of expert information, and can also use other fuzzy processing methods to obtain different types of weight decision analysis processes.

The fuzzy group analytic hierarchy process (FDAHP) discussed in this paper has been compiled into a spreadsheet-based software, and interactive decision analysis can be easily carried out by means of Microsoft Excel.

\section{References}

[1] Saaty, T. L., The Analytic Hierarchy Process: Planning, Priority Setting. New York: McGraw-Hill: Resource Allocation, 1980

[2] Wang Lianfen, Xu Shubai. Introduction to analytic hierarchy process [ M ]. Beijing: People's University of China Press, 1990.

[3] Corvin Jaime A et al. Analytic hierarchy process: An innovative technique for culturally tailoring evidencebased interventions to reduce health disparities.[J]. Health expectations: an international journal of public participation in health care and health policy, 2021, 24 Suppl 1: 70-81.

[4] Li Chunhao, Du Yuanwei, Sun Yonghe, Tian Shuo. Multiple Attribute Implicit Variable Weight Decision Analysis Method [ J ]. China Management Science, 2012, 20 ( 05 ) : 163-172.

[5] Zhou Lianchun and Jinshan Zhang and Konrad Fröhling. Evaluation of coal mine pollution abatement benefit based on analytic hierarchy process [J]. Arabian Journal of Geosciences, 2020, 13(19) : 71-86.

[6] Wang Xuejun, Guo Yajun. Consistency analysis of judgment matrix based on G1 method [ J ]. Chinese management science, 2006 ( 03 ) : 65-70.

[7] Lu Yuejin. Ranking of fuzzy analytic hierarchy process based on fuzzy consistent matrix [ $\mathrm{J}$ ]. Fuzzy systems and mathematics, 2002, $16(2): 79-85$.

[8] Qin Guangyu et al. Comprehensive evaluation of regional energy internet using a fuzzy analytic hierarchy process based on cloud model: A case in China[J]. Energy, 2021, 228.

[9] Zhang Jijun. Fuzzy Analytic Hierarchy Process ( FAHP ) [ J ].Fuzzy System and Mathematics, 2000,14 ( 2 ) : 80-88.

[10] Singh Rajbala and Kumar Deepak and Sagar Bharat Bhushan. Fuzzy analytical hierarchical process based two way assessment for agile testing[J]. Journal of Discrete Mathematical Sciences and Cryptography, 2021, 24(3): 745-762.

[11] Tang Youwen. Fuzzy Analytic Hierarchy Process [ J ]. Journal of Qinghai Normal University ( Natural Science Edition ), 2002, ( 3 ): 19-23.

[12] Tao Shehui. How to construct fuzzy consistent matrix in fuzzy analytic hierarchy process [ $\mathrm{J}$ ].Journal of Sichuan Normal University ( Natural Science Edition ), 2002, 23 ( 3 ) : 282-285. 